\title{
Patterns of recurrence and incidence of second primary tumors after lobectomy by means of video-assisted thoracoscopic surgery (VATS) versus thoracotomy for lung cancer
}

\author{
Raja M. Flores, MD, ${ }^{\mathrm{a}}$ Ugonna N. Ihekweazu, MD, ${ }^{\mathrm{b}}$ Nabil Rizk, MD, ${ }^{\mathrm{b}}$ Joseph Dycoco, BA, \\ Manjit S. Bains, MD, ${ }^{\mathrm{b}}$ Robert J. Downey, MD, ${ }^{\mathrm{b}}$ Prasad Adusumilli, MD, ${ }^{\mathrm{b}}$ David J. Finley, MD, ${ }^{\mathrm{b}}$ \\ James Huang, MD, ${ }^{\mathrm{b}}$ Valerie W. Rusch, MD, ${ }^{\mathrm{b}}$ Inderpal Sarkaria, MD, ${ }^{\mathrm{b}}$ and Bernard Park, $\mathrm{MD}^{\mathrm{b}}$
}

Objective: Reports have questioned the oncologic efficacy of video-assisted thoracoscopic surgery when compared with thoracotomy despite similar survival results. In response, we investigated the pattern of recurrent disease and the incidence of second primary tumors after lobectomy by means of video-assisted thoracoscopic surgery and thoracotomy.

Methods: All patients who underwent lobectomy for clinical stage IA lung cancer determined by means of computed tomographic and positron emission tomographic analysis were identified from a prospective database at a single institution. All patients were selected for video-assisted thoracoscopic surgery or thoracotomy by an individual surgeon. Patients' characteristics, perioperative results, recurrences, and second primary tumors were recorded. Variables were compared by using Student's $t$ test, the Pearson $\chi^{2}$ test, and Fisher's exact test. A logistic regression model was constructed to identify variables influencing the development of recurrent disease or metachronous tumors.

Results: From 2002 to 2009, 520 patients underwent lobectomy by means of video-assisted thoracoscopic surgery, and 652 underwent lobectomy by means of thoracotomy. Final pathological stage was similar in the video-assisted thoracoscopic surgery and thoracotomy groups. Logistic regression demonstrated a lower risk (odds ratio, $0.65 ; P=.01$ ) of recurrent disease in patients undergoing video-assisted thoracoscopic surgery after adjusting for age, stage, sex, histology, tumor location, and synchronous primary tumors.

Conclusions: Recurrence rates for video-assisted thoracoscopic surgery appear to be at least equivalent to those for thoracotomy. This study supports lobectomy by means of video-assisted thoracoscopic surgery as an oncologically sound technique. (J Thorac Cardiovasc Surg 2011;141:59-64)

The oncologic efficacy of lobectomy by means of videoassisted thoracoscopic surgery (VATS) compared with thoracotomy for lung cancer remains controversial despite studies demonstrating similar survival results. ${ }^{1,2}$ Those who advocate only thoracotomy believe VATS might leave unrecognized tumor within lymph node drainage areas and fissure staple lines and at the bronchial margins, can cause pleural tumor seeding from lung manipulation, and might fail to identify second primary cancers because of the lack of bimanual lung palpation. ${ }^{3}$

Large case series and comparative studies use survival as the primary end point in determining the adequacy of VATS

\footnotetext{
From the Division of Thoracic Surgery, ${ }^{a}$ Mount Sinai School of Medicine, and the Department of Surgery, ${ }^{\mathrm{b}}$ Thoracic Service, Memorial Sloan-Kettering Cancer Center, New York, NY.

Disclosures: Authors have nothing to disclose with regard to commercial support.

Read at the 90th Annual Meeting of The American Association for Thoracic Surgery, Toronto, Ontario, Canada, May 1-5, 2010.

Received for publication April 30, 2010; revisions received Aug 11, 2010; accepted for publication Aug 24, 2010; available ahead of print Nov 9, 2010.

Address for reprints: Raja M. Flores, MD, Division of Thoracic Surgery, Mount Sinai

School of Medicine, 1190 Fifth Ave, Box 1028, New York, NY 10029 (E-mail: raja.

flores@mountsinai.org).

$0022-5223 / \$ 36.00$

Copyright (c) 2011 by The American Association for Thoracic Surgery

doi:10.1016/j.jtcvs.2010.08.062
}

lobectomy. ${ }^{1,2}$ However, survival results short of a prospective randomized controlled trial are subject to significant flaws in methodology and selection bias. Thoracic cooperative groups are not enthusiastic to perform prospective randomized controlled trials of sufficient power because of accrual concerns, lack of technique uniformity, and surgeon's bias. Although prolonged survival is the ultimate goal of most oncologic interventions, pattern of recurrence is another reasonable measure of oncologic efficacy. We undertook this study to investigate any differences in the patterns of recurrence after lobectomies performed by means of VATS versus thoracotomy for lung cancer.

\section{MATERIALS AND METHODS \\ Data Acquisition}

All patients with clinical stage IA lung cancer determined by means of computed tomographic and positron emission tomographic scanning who subsequently underwent resection by means of lobectomy were identified from a prospectively maintained institutional thoracic surgical database after institutional review board approval. Excluded patients included those with a history of preoperative chemotherapy; histologic diagnosis in the primary lesion of benign disease; or procedures other than a lobectomy for the primary lesion, such as exploration only or wedge, segmentectomy, bilobectomy, pneumonectomy, or chest wall resection. Patients who also 


\section{Abbreviation and Acronym \\ VATS $=$ video-assisted thoracoscopic surgery}

received an additional wedge resection or segmentectomy for a synchronous primary tumor were included.

Variables recorded included age, sex, tumor size, nodal status, final pathological stage, histology, and follow-up status. Locoregional recurrences were defined as tumor recurrence at the staple line, bronchial stump, ipsilateral pleura, nodal drainage areas, or ipsilateral lung within 2 years of resection. Distant recurrences were defined as tumor recurrence at a site distant from the original operation, including contralateral lung, brain, liver, bone, disseminated, and other. Second primary tumors were defined from a modification of the Martini and Melamed criteria as (1) tumors of different histologies, (2) tumors of different histologic subtypes, (3) tumors of similar histology but arising from separate foci, and (4) tumors of similar histology in the absence of metastatic disease in intervening regional or mediastinal lymph node stations in the absence of extrathoracic disease. ${ }^{4,5}$

\section{Operative Technique}

The decision to perform either VATS or thoracotomy was made by the individual surgeon. Eight surgeons (R.M.F., V.W.R., B.P., N.R., P.A., J.H., D.J.F., and I.S.) perform VATS lobectomy for early-stage patients, whereas 2 surgeons (R.J.D. and M.S.B.) exclusively perform thoracotomy and lobectomy for such patients.

All patients underwent standard anesthesia with single-lung ventilation, low intraoperative airway pressures, and perioperative fluid restriction. Postoperative pain relief was provided by continuous epidural administration of fentanyl and bupivacaine, intravenous opioid administration, or both.

VATS lobectomy was performed through a 4-cm utility incision at the anterior axillary line at the third or fourth interspace by using standard thoracic instruments without rib spreading, a 2-cm anterior thoracostomy port at the eighth interspace at the anterior axillary line for the camera, and a 2-cm posterior port for retraction and stapler insertion. The operation was performed entirely under thoracoscopic visualization. The hilar structures were individually ligated by endoscopic staplers, and a mediastinal nodal dissection or sampling was performed. The camera port was subsequently used as a thoracostomy tube site. Our technique has been described previously. ${ }^{6-8}$ In VATS cases in which robotic assistance was used for dissection, the same 3 VATS incisions were used as described in an earlier report. ${ }^{9}$

Lobectomy by means of thoracotomy was performed through a posterolateral thoracotomy incision, which spared the serratus anterior muscle. The chest was entered through the fifth interspace, and a Finochietto retractor was used to gain exposure. Endoscopic staplers were routinely used for the transection of vessels and the completion of the fissures. In all patients an ipsilateral mediastinal dissection or sampling was performed.

\section{Statistical Methods}

Patients' characteristics and perioperative data were compared by using Student's $t$ test, the Pearson $\chi^{2}$ test, and Fischer's exact test. The KaplanMeier method and log-rank test were used to determine time to recurrence and statistical significance, respectively. STATA 10 software (StataCorp, College Station, Tex) was used to perform statistical analyses.

\section{RESULTS}

From 2002 to 2009, 520 patients underwent lobectomy by means of VATS, and 652 underwent lobectomy by means of thoracotomy. Patients' characteristics are demonstrated in Table 1. There were 2 perioperative deaths in each group,
TABLE 1. Patients' characteristics

\begin{tabular}{|c|c|c|c|}
\hline & $\begin{array}{c}\text { VATS } \\
\text { group } \\
(\mathbf{n}=\mathbf{5 2 0}) \\
\end{array}$ & $\begin{array}{c}\text { Thoracotomy } \\
\text { group } \\
(\mathbf{n}=\mathbf{6 5 2}) \\
\end{array}$ & $\begin{array}{c}P \\
\text { value } \\
\end{array}$ \\
\hline Age $(y)$ & 67 & 67 & .8 \\
\hline Female sex & $331(64 \%)$ & $402(62 \%)$ & .5 \\
\hline Size $(\mathrm{cm})$ & 2.1 & 2.1 & .8 \\
\hline \multicolumn{4}{|l|}{ Stage } \\
\hline IA & $344(66 \%)$ & $420(65 \%)$ & .2 \\
\hline IB & $92(18 \%)$ & $94(14 \%)$ & \\
\hline IIA & $24(5 \%)$ & $36(6 \%)$ & \\
\hline IIB & $18(3 \%)$ & $28(4 \%)$ & \\
\hline IIIA & $31(6 \%)$ & $47(7 \%)$ & \\
\hline IIIB & $11(2 \%)$ & $27(4 \%)$ & \\
\hline \multicolumn{4}{|l|}{ Primary tumor location } \\
\hline RUL & $195(38 \%)$ & $237(36 \%)$ & .3 \\
\hline RML & $33(6 \%)$ & $51(8 \%)$ & \\
\hline RLL & $90(17 \%)$ & $103(16 \%)$ & \\
\hline LUL & $145(28 \%)$ & $170(26 \%)$ & \\
\hline LLL & $57(11 \%)$ & $91(14 \%)$ & \\
\hline \multicolumn{4}{|l|}{ Histology } \\
\hline Adenocarcinoma & $190(37 \%)$ & $220(34 \%)$ & .03 \\
\hline Adenocarcinoma with BAC & $219(42 \%)$ & $239(37 \%)$ & \\
\hline Squamous cell carcinoma & $55(11 \%)$ & $79(12 \%)$ & \\
\hline Large cell carcinoma & $21(4 \%)$ & $42(6 \%)$ & \\
\hline Small cell carcinoma & $4(0.01 \%)$ & $6(0.01 \%)$ & \\
\hline $\mathrm{BAC}$ & $4(0.01 \%)$ & $16(.02 \%)$ & \\
\hline Carcinoid & $27(5 \%)$ & $50(8 \%)$ & \\
\hline
\end{tabular}

VATS, Video-assisted thoracoscopic surgery; $R U L$, right upper lobe; $R M L$, right middle lobe; $R L L$, right lower lobe; $L U L$, left upper lobe; $L L L$, left lower lobe.

and median follow-up was 25 months in the thoracotomy group and 23 months in the VATS group. The groups were similar with respect to age, sex, size of the primary tumor, distribution of pathological stage, and tumor location. There was a slightly higher proportion of adenocarcinoma in the VATS group $(79 \%$ vs $71 \%, P=.03)$.

There was a significantly higher number of recurrences in the thoracotomy group than in the VATS group, as demonstrated in Table 2. This appears to be predominantly secondary to a higher rate of distant recurrence in the thoracotomy group $(11 \%$ vs $6 \%, P<.0001)$. More importantly, the rate of locoregional recurrence between the 2 groups was identical. Synchronous primary tumors identified at the initial operation were $34(7 \%)$ by means of VATS and $78(12 \%)$

TABLE 2. Patterns of recurrence and metachronous lesions $(\mathbf{n}=\mathbf{1 1 7 2})$

\begin{tabular}{llcl}
\hline & $\begin{array}{c}\text { VATS group } \\
(\mathbf{n}=\mathbf{5 2 0})\end{array}$ & $\begin{array}{c}\text { Thoracotomy group } \\
(\mathbf{n}=\mathbf{6 5 2})\end{array}$ & $\boldsymbol{P}$ value \\
\hline Locoregional & $20(4 \%)$ & $33(5 \%)$ & \\
Distant & $33(6 \%)$ & $70(11 \%)$ & \\
Metachronous & $15(3 \%)$ & $21(3 \%)$ & \\
Total & $68(13 \%)$ & $124(19 \%)$ & $<.001$ \\
\hline
\end{tabular}

VATS, Video-assisted thoracoscopic surgery. 
TABLE 3. Patterns of recurrence and metachronous lesions excluding synchronous primary tumors $(n=1058)$

\begin{tabular}{lccc}
\hline & $\begin{array}{c}\text { VATS group } \\
(\mathbf{n}=\mathbf{4 8 6})\end{array}$ & $\begin{array}{c}\text { Thoracotomy group } \\
(\mathbf{n}=\mathbf{5 7 2})\end{array}$ & $\begin{array}{c}\boldsymbol{P} \\
\text { value }\end{array}$ \\
\hline Locoregional & $18(3 \%)$ & $29(5 \%)$ & \\
Distant & $32(7 \%)$ & $63(11 \%)$ & \\
Metachronous & $13(3 \%)$ & $14(3 \%)$ & $<.01$ \\
Total & $63(13 \%)$ & $106(19 \%)$ & \\
\hline \multicolumn{2}{l}{ VATS, Video-assisted thoracoscopic surgery. }
\end{tabular}

by means of thoracotomy $(P=.01)$. All patients with synchronous primary tumors underwent an additional wedge resection or a segmentectomy, and all patients received $\mathrm{R} 0$ resections. Because of the concern that synchronous disease found at the time of initial exploration instead represented advanced disease, a similar analysis was performed after excluding patients with synchronous primary tumors (Table 3). The pattern of recurrence remained the same, and in particular, the rate of distant recurrence in the thoracotomy group remained higher than that in the VATS group.

A logistic regression model was then constructed with the development of any recurrences or metachronous tumors as the dependent variable, controlling for age, sex, stage, histology, size, and synchronous primary tumors. VATS demonstrated a lower rate of recurrence (hazard ratio, 0.65; $P<.01$; Table 4).

Time to recurrence was then evaluated by using the Kaplan-Meier failure function, as demonstrated in Figure 1. The rate of recurrence of VATS was 0.09 per patient per year, whereas that of thoracotomy was 0.12 per patient per year $(P=.06, \log$-rank test $)$.

\section{DISCUSSION}

VATS lobectomy was first performed nearly 2 decades ago, but only $16 \%$ of lobectomies in the United States are currently performed with this method. ${ }^{10,11}$ Initial concerns of bleeding and operative mortality have been dispelled by multiple large case series demonstrating satisfactory perioperative outcomes. ${ }^{1,2,10,12,13}$ The Cancer and Leukemia Group B demonstrated the feasibility of performing VATS lobectomy at different institutions, but several randomized controlled trials have been too underpowered to draw any meaningful conclusions with regard to survival. ${ }^{2,14-16}$

TABLE 4. Logistic regression of any recurrence or metachronous cancer

\begin{tabular}{llc}
\hline & OR & $\boldsymbol{P}$ value \\
\hline Age & 1.02 & .01 \\
Sex (female) & 0.89 & .5 \\
Pathological stage (stage $>1)$ & 2.6 & $<.0001$ \\
VATS & 0.65 & .01 \\
Synchronous primary tumors & 1.3 & .3 \\
Histology (adenocarcinoma) & 1.03 & .3 \\
\hline
\end{tabular}

$O R$, Odds ratio; VATS, video-assisted thoracoscopic surgery.

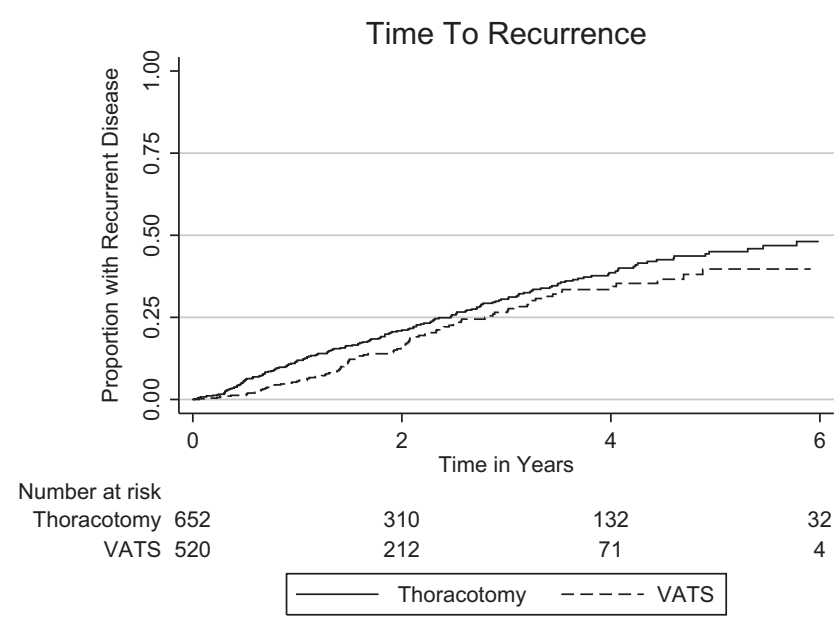

FIGURE 1. Time to recurrence. VATS, Video-assisted thoracoscopic surgery.

Oncologic concerns remain in the thoracic community despite several well-balanced comparative studies demonstrating equivalent survival. ${ }^{1-3}$ However, no study to date has evaluated recurrence as the primary end point.

A major limitation of previous studies has been an imbalance in stage distribution between the VATS and thoracotomy groups. ${ }^{2,17,18}$ Stage migration is inevitable. All patients in this study were selected based on clinical stage IA disease, but more importantly, the final pathological stage distribution was identical in both groups. This is a major strength because pathological stage was the most significant predictor of recurrent disease in the multivariate model (odds ratio, 2.6; $P<.0001$ ). The pattern of locoregional recurrences was similar between the 2 groups. The greatest difference between the 2 groups resulted from a higher rate of distant recurrences in the thoracotomy group. Based on the given data, the conclusion that VATS provides local control at least equivalent to that provided by thoracotomy appears justified.

A recent study raised concerns that VATS might miss small lesions at the time of the initial operation. ${ }^{3}$ This study investigated the number of lesions not apparent on preoperative computed tomographic scans that were identified at the time of thoracotomy by means of bimanual palpation and found that $8.4 \%$ of patients were identified as having another cancerous nodule. The study made a critical but invalid assumption that VATS would miss all these small lesions. Consequently, the final conclusion of the study was not justified because not a single patient actually underwent VATS exploration or resection. Experienced VATS surgeons, in contrast, believe digital palpation at the time of initial resection is sufficient to identify synchronous lesions. Therefore we investigated the influence of synchronous primary tumors resected at the time of lobectomy because these might represent more advanced cancer that is not captured by stage alone. The thoracotomy group demonstrated 
a greater number of synchronous lesions than the VATS group ( $12 \%$ vs $7 \%$, respectively). To explore further, we also performed a separate analysis with synchronous primary tumors excluded and found the same pattern of recurrence. We hypothesized that if VATS missed synchronous primary tumors at the time of initial lobectomy, then the VATS group should demonstrate a greater number of metachronous tumors over time. Surprisingly, the number of metachronous lesions that developed in each group was similar. Interestingly, the influence of synchronous primary tumors as a potential confounder on outcome has not been addressed in the vast majority of published VATS studies. Based on the results of this study, the influence does not appear to be substantial because the presence of synchronous primary tumors in the multivariate model was not significant (odds ratio, 1.3; $P=.3$ ).

The role of histology in non-small cell lung cancer has been studied extensively. The vast majority of recent studies demonstrate that cancer recurrences and cancer-related deaths are somewhat greater in patients with adenocarcinoma than other non-small cell histologies. ${ }^{19,20}$ The VATS group in our study demonstrated a greater number of patients with adenocarcinoma and a lower number of patients with carcinoid tumor, which would appear to favor the thoracotomy group. However, the results demonstrated the contrary.

A major limitation of this study is selection bias. The comparisons in this study are inextricably confounded by the individual surgeon's practice: $100 \%$ of the VATS lobectomies are performed by 8 surgeons, whereas 2 surgeons only perform thoracotomies. Thus unrecorded biases cannot be separated from those related to the specific technique. Surgeon bias might have led to more synchronous primary tumors in the thoracotomy group and more adenocarcinomas in the VATS group. The 2-month difference in length of follow-up could result in a slight bias in favor of the VATS group. There appears to be an immeasurable factor or selection bias that consistently leads to improved outcome in patients undergoing VATS observed with many studies, or there might be a potential biological explanation related to less trauma translating into an advantage. ${ }^{21}$

Thoracotomy is accepted as the gold standard in lung cancer surgery. The pattern of recurrence demonstrated in this study supports the argument that VATS lobectomy is at least equivalent to the gold standard. However, no study can replace clinical judgment at the time of the operation. In situations in which oncologic principles might be compromised, VATS should be converted to a thoracotomy. However, in appropriately selected patients VATS lobectomy is an oncologically sound procedure.

We thank Dr Rob McKenna for allowing Dr Raja Flores to observe his operative technique. Also, we thank Erlin Daley for her editorial assistance.

\section{References}

1. Flores RM, Park BJ, Dycoco J, Aronova A, Hirth Y, Rizk NP, et al. Lobectomy by video-assisted thoracic surgery (VATS) versus thoracotomy for lung cancer. J Thorac Cardiovasc Surg. 2009;138:11-8.

2. Flores RM, Alam N. Video-assisted thoracic surgery lobectomy (VATS), open thoracotomy, and the robot for lung cancer. Ann Thorac Surg. 2008;85(suppl): S710-5.

3. Cerfolio RJ, Bryant AS. Is palpation of the nonresected pulmonary lobe(s) required for patients with non-small cell lung cancer? A prospective study. J Thorac Cardiovasc Surg. 2008;135:261-8.

4. Martini N, Melamed MR. Multiple primary lung cancers. J Thorac Cardiovasc Surg. 1975;70:606-12

5. Finley DJ, Yoshizawa A, Travis W, Zhou Q, Seshan VE, Bains MS, et al. Predictors of outcomes after surgical treatment of synchronous primary lung cancers. J Thorac Oncol. 2010;5:197-205.

6. Flores RM. VATS lobectomy: focus on technique. World J Surg. 2010;34:616-20.

7. Flores RM, VATS lobectomy for early stage lung cancer. CTSNET experts' techniques. Available at: http://www.ctsnet.org/sections/clinicalresources/ thoracic/expert_tech-.html.

8. Weyant MJ, Flores RM. VATS mediastinal nodal dissection. Available at: http:// www.ctsnet.org/sections/clinicalresources/thoracic/expert_tech-26.html.

9. Park BJ, Flores RM, Rusch VW. Robotic-assisted VATS lobectomy: development of a uniform technique and initial results. J Thorac Cardiovasc Surg. 2006;131:54-9.

10. McKenna RJ, Houck W, Fuller CB. Video-assisted thoracic surgery lobectomy: experience with 1,100 cases. Ann Thorac Surg. 2006;81:421-6.

11. Boffa DJ, Allen MS, Grab JD, Gaissert HA, Harpole DH, Wright CD. Data from the Society of Thoracic Surgeons General Thoracic Surgery database: the surgical management of primary lung tumors. J Thorac Cardiovasc Surg. 2008;135: 247-54.

12. Onaitis MW, Petersen RP, Balderson SS, Toloza E, Burfeind WR, Harpole DH Jr, et al. Thoracoscopic lobectomy is a safe and versatile procedure: experience with 500 consecutive patients. Ann Surg. 2006;244:420-5.

13. Walker WS, Codispoti M, Soon SY, Stamenkovic S, Carnochan F, Pugh G. Longterm outcomes following VATS lobectomy for non-small cell bronchogenic carcinoma. Eur J Cardiothorac Surg. 2003;23:397-402.

14. Swanson SJ, Herndon JE, D'Amico TA, Demmy TL, McKenna RJ, Green MR, et al. Video-assisted Thoracic surgery lobectomy: Report of CALGB 39802a prospective, multi-institution feasibility study. J Clin Oncol. 2007;25:4993-7.

15. Yim AP, Landreneau RJ, Izzat MB, Fung AL, Wan S. Is video-assisted thoracoscopic lobectomy a unified approach? Ann Thorac Surg. 1998;66:1155-8.

16. Kirby TJ, Mack MJ, Landreneau RJ, Rice TW. Lobectomy-video-assisted thoracic surgery versus muscle-sparing thoracotomy: a randomized trial. J Thorac Cardiovasc Surg. 1995;109:997-1002.

17. Watanabe A, Koyanagi T, Ohsawa H, Mawatari T, Nakashima S, Takahashi N, et al. Systematic node dissection by VATS is not inferior to that through an open thoracotomy: a comparative clinicopathologic retrospective study. Surgery. 2005;138:510-7.

18. Whitson BA, Andrade RS, Boettcher A, Bardales R, Kratzke RA, Dahlberg PS, et al. Video-assisted thoracoscopic surgery is more favorable than thoracotomy for resection of clinical stage I Non-small cell lung cancer. Ann Thorac Surg. 2007;83:1965-70.

19. Thomas PA, Rubinstein L. Malignant disease appearing late after operation for T1 N0 non-small cell lung cancer. The Lung Cancer Study Group. J Thorac Cardiovasc Surg. 1993;106:1053-8.

20. Okada M, Nishio W, Sakamoto T, Harada Uchino K, Tsubota N. Long term survival and prognostic factors of five-year survivors with complete resection of non-small cell lung carcinoma. J Thorac Cardiovasc Surg. 2003;126:558-62.

21. Craig SR, Leaver HA, Yap PL, Pugh GC, Walker WS. Acute phase responses following minimal access and conventional thoracic surgery. Eur J Cardiothorac Surg. 2001;20:455-63.

\section{Discussion}

Dr Scott Swanson (Boston, Mass). Raja, you and your colleagues are to be congratulated for a very nice study looking at an important issue in lung cancer: that of recurrence following resection for early-stage disease. Your manuscript, which you kindly provided me, is well written. 
You and your colleagues had excellent outcomes with an extremely low operative mortality and a local recurrence rate of about $5 \%$, which is similar to our published recurrence rate of about $3 \%$. Again, similar to your other papers for early clinical stage disease was a finding of about $1 / 3$ of patients being clinically understaged, which reminds us all of the relatively low sensitivity of our radiologic imaging, even with the advent of expensive and relatively routine positron emission tomography scans. Also, you had about $10 \%$ of patients with stage IIIA or IIIB disease. First question, can you tell us how many patients had cervical mediastinoscopy?

Dr Flores. I don't know how many patients had cervical mediastinoscopy.

Dr Swanson. Did any of the patients have that before their lobectomy?

Dr Flores. If the positron emission tomography scan is positive, the practice varies among surgeons. Some will do mediastinoscopy, some will send the patients for induction chemotherapy, and some will just resect and give chemotherapy afterward.

Dr Swanson. But of these 1100 clinical stage IA, did some have a preresectional mediastinoscopy?

Dr Flores. I'm sure some. I think the number is relatively small. This was all for clinical stage IA disease, so I'd have to say the number is very, very small.

Dr Swanson. Okay. In the manuscript, you report that your data was kept in a prospectively maintained database. Can you tell us how this is prospectively maintained, what percentage of your patients had follow-up, and do you use the Society of Thoracic Surgeons database?

Dr Flores. We are members of the Society of Thoracic Surgeons database. This is prospectively maintained. Every Friday morning, we have a conference where the attending is present, we go over the staging, the attending gives their input as to what was identified at the time of surgery, and this is recorded by a single research assistant.

Dr Swanson. A number of patients were found to have synchronous primaries-about $10 \%$. Were these suspected based on the preoperative computed tomography or were they complete surprises?

Dr Flores. That's a difficult question to answer. I think it's a very good question and something that I tried to look into. From the database, it's difficult to tell how many of these were planned and how many of these were incidentally found. The majority of them were less than a centimeter. Most likely they were incidentally found. I can't ascertain that from the database.

Dr Swanson. Finally, most striking about this paper is the statistically significant decrease in overall recurrence for video-assisted thoracoscopic surgery (VATS) versus open lobectomy, particularly because the stage distribution was the same and, more importantly, the size was identical between the 2 groups$2.1 \mathrm{~cm}$ - and there were more adenocarcinomas in the VATS group. This mirrors the findings of a large meta-analysis by the Minnesota group that I was involved with and published in the $A n$ nals of Thoracic Surgery last year. What do you make of this, and do you think this is related to the concept of less operative trauma and suppression of the immune system? With such a striking result, about a $6 \%$ difference, which would buy somebody adjuvant chemotherapy, do you think patients should be recommended to have a VATS procedure for oncologic reasons?
Excellent work, Raja, and I appreciated the chance to comment on the paper.

Dr Flores. Thank you.

I think whenever it comes to recommending a surgical procedure because of biologic reasons, and many have published on that, about decreased cytokines, interleukins, that could have a favorable oncologic outcome on the patient, I think we have to be careful about jumping to conclusions with that. I am still not convinced based on the nature of this study that there is not a selection bias going on, which is immeasurable. We did not anticipate having a higher number of synchronous primaries in the thoracotomy group. Of course, after further investigation, we identified that. That is also something that I think many of the previous VATS papers don't comment on. So I'm sure that there is some selection bias taking place that favors the VATS patients. I just can't figure it out. I hope there is a potential to figure out some underlying biologic advantage from less trauma, but I don't think I would make that statement based on this data.

Dr Bryan Meyers (St. Louis, Mo). You can eliminate the possibility of a selection bias by doing some sort of a propensity matching. That might be a help. You can also discuss briefly the possibility of a detection bias; if only 2 surgeons were doing the thoracotomy and 8 were doing the VATS, do they have a different protocol for following these patients up that might have led to a difference? And then the other thing which was interesting, kind of a coincidence, when we were quizzing Bob Timmerman at the General Thoracic Surgical Club about the low rate of recurrence after stereotactic radiation therapy, based on what we know exists if you do a lymph node dissection in N1 and N2 stations in clinical stage I lung cancer, there was a suggestion or hint that maybe the decreased hit on the immune system that stereotactic radiation therapy has helps reduce the chances of recurrence over VATS lobectomy. So, if you think VATS lobectomy is better because it's less invasive, then you might have to take the next step.

Dr Flores. Well, as a thoracic surgeon, I refuse to accept that. I think the good question is the propensity score. I investigated whether we should do propensity score analysis on this paper or not. We did it on our last paper. I think when you have a cohort that is as well balanced as this, it may actually make it worse, and it did make it worse on our last paper. Propensity score analyses are best when it's performed on thousands of patients. When you have hundreds of patients, such as this, I can argue that it may not be as good, and it throws out a lot of useful data when you narrow down your cohort to that extent.

Dr Mark Krasna (Baltimore, $M d$ ). Great paper, Raja. Because you have such a very good cohort in the 7-year time frame, you have another unique opportunity related to what Dr Meyers hinted to, and that is follow-up. So, can you tell us what the current routine follow-up is at Sloan for your patients during that 7-year period? One of the very interesting things that you found was no specific difference in your metachronous lesions. You did talk about a difference in distant failure, but it would actually be very interesting to see was there a difference in terms of the metachronous lesions with VATS lobe versus open. So did you follow the same follow-up routine for all 8 surgeons over the 7 years?

Dr Flores. All surgeons follow-up patients the same way. I'm sorry, Bryan, I forgot to address that.

Dr Krasna. So what is that algorithm? 
Dr Flores. Basically, computerized axial tomography scans after surgery every 6 months.

Dr Krasna. For how many years?

Dr Flores. I follow my patients forever. I think most of the attendings will get computerized axial tomography scans once a year. It's every 6 months, and then when you hit 5 years, once a year.

Dr Cerfolio. You follow every patient forever? God bless you.

Dr Thomas D'Amico (Durham, NC). Raja, you explained that there were 8 surgeons that did VATS, 2 that didn't, and yet less than $50 \%$ of the total clinical stage IA were VATS. You would have thought it would be $80 \%$. So there had to be some selection. How did it get to be less than $50 \%$ ? That's the first question. And how did you analyze your conversions?

Dr Flores. Well, the majority of surgeons who do the bulk of the cases, the VATS cases and the open cases, have bigger practices than the younger guys who are just starting, so that's where the majority of cases come from. As far as conversions, this is something that I have labored over with this study, and that's a point that I always bring up, and in our last paper that was one of the center points: What do you do with the conversions. In my last paper, because survival was the primary endpoint, I included them in an intent-to-treat analysis in the VATS group. In this study recurrence rate is the primary endpoint. I included those in the thoracotomy group for a variety of reasons, including consultation with 6 biostatisticians to be sure that that was appropriate for this study. So that's usually my question. For this study, because the gold standard is thoracotomy and because of the lack of bimanual palpation in the VATS group, we thought that it was better to analyze this group in the thoracotomy group.

Dr Sandro Mattioli (Bologna, Italy). First, what kind of thoracotomy did you do? Second, did you consider the position of the small nodule?
Dr Flores. We looked at the location of the nodule, you know, in the upper, lower, middle lobe. We didn't look specifically within that lobe. The thoracotomy was usually a serratus-sparing thoracotomy, fifth interspace, and a Finochietto retractor was used in the majority of cases.

Dr Joseph Shrager (Stanford, Calif). You didn't emphasize it in your presentation, but in the abstract you emphasized that the synchronous primary rate would be much higher at thoracotomy. However, I would argue that that's basically not relevant because presumably the patients that have a suspected synchronous primary, a little 5-mm ground-glass opacity or something, on the preoperative scan, are most often going to get a thoracotomy, unless it's clearly accessible to VATS - very peripheral.

Dr Flores. I'm not sure if I agree with that. I've tried to figure out how to explain this, and there is no way of knowing ahead of time. When you look at Cerfolio's paper, and that actually prompted me to perform this, the number of malignancies that he described in his paper was $8.4 \%$. Ours is $7 \%$. Our thoracotomy group was $11 \%$. I think it's tough to tell what we're doing before we get to the operating room based on what's recorded in the database.

Dr Shrager. I suspect that most people who do these operations both ways will tend to do an open operation if, in terms of second lesions, there's anything more than one very peripheral one.

Dr Flores. Not me. If there are 2 lesions there, I'll go ahead with the lobectomy for the larger lesion and I'll wedge or segment out the second lesion.

Dr Shrager. You can't always find a 5-mm ground-glass lesion with your finger at VATS- unless it's very peripheral.

Dr Flores. Most of the time with digital palpation, I'll argue that we can. 